\title{
Unsuccessful A ttempt at Reversing Intractable Ventricular Arrhythmia in a Post CABG Patient
}

\author{
Masoom Siraj, ${ }^{1}$ M d. Hamidur Rahman, ${ }^{2} \mathrm{~F}$ arzana Yasmin ${ }^{3}$
}

\section{ABSTRACT}

The case of a 58 years old, gentleman is described here who underwent an emergency Coronary A rtery Bypass Grafting (CA B G) at Ibrahim Cardiac Hospital \& Research Institute. Right after the procedure he started developing ventricular arrhythmias. We present here the various challenges that we had to face and the options available to us in the setting of a third world hospital to manage a case of intractable ventricular arrhythmia. The patient underwent revascularization, intra-aortic balloon pump support, pharmacological manipulation, burst pacing with over drive suppression and cardio-version. Yet we were unable to control the fatal ventricular arrhythmia with an unfavorable outcome. Our case stands out from the rest as arrhythmia started after revascularization as opposed to many a case that is treated by revascularization

\section{Introduction}

Ventricular arrhythmia following Coronary A rtery Bypass Surgery is a known complication, whether in the form of Ventricular Tachycardia (VT) or Ventricular Fibrillation (VF). This is more so in a heart with low Ejection Fraction (EF). ${ }^{1}$ If $V T$ repeatedly recurs despite optimal medical management and does not go away for more than 12 hours of a 24 hour period, it is designated as Incessant. It is also defined as Intractable Ventricular Tachy-A rrhythmia (IVTA) when frequent, recurrent and recalcitrant ventricular tachy-arrhythmias result in significant symptoms and haemodynamic compromise. ${ }^{2}$ IVTA is associated with poor outcome. ${ }^{3}$ This arrhythmia may be due to abnormal reentry circuits originating from an area of ischaemic scar. Damage or fibrosis of the myocardium or stretch in dilated hearts can cause these abnormal circuits. During cardiac surgery shocking out a fibrillating heart is a common thing and most often this is all that needs to be done. Sometimes an anti-arrhythmia drug is needed during the post surgical period. Problems arise when the usual ventricular arrhythmias take the form of IVTA. In our case the patients went through all the probable ways of dealing with an IVTF excepting the very costly modalities of Implantable CardioDefibrillators (ICD), Extra Corporeal M embrane Oxygenation (ECM O) followed by cardiac transplant.

\section{C ase history}

A 58 years old male patient was admitted to the CCU of Ibrahim Cardiac Hospital \& Research Institute with the complaints of shortness of breath for the last three days. He was diagnosed as a case of late presentation anterior myocardial infarction (AMI) with acute left ventricular failure (ALVF).

On admission pulse was 124 / min and was regular in rhythm, systolic and diastolic blood pressures were 210 and $110 \mathrm{mmHg}$ respectively, coarse crepitations on auscultation in the lungs on both sides, ECG showed ST elevation with pathological Q waves in V 1-V 5 leads, Troponin-I 2.44, serum creatinine $1.48 \mathrm{mg} / \mathrm{dl}$, Echo revealed regional wall motion abnormality, an ejection fraction of $30 \%$, Grade-II mitral regurgitation (MR) and mild pulmonary hypertension.

Initially he was stabilized with medical management in particular for LVF. Coronary A ngiogram (CA G) done 10 days after admission revealed $100 \%$ occlusion of the left anterior descending (LAD) artery with retrograde filling, and critical lesion in non-dominant right coronary artery (RCA). A ttempted percutaneous coronary intervention $(\mathrm{PCI})$ to $L A D$ failed. The patient was shifted back to CCU and emergency CABG was done on the next day to establish blood flow to the occluded LAD. During the procedure dense adhesions were found between pericardium and the epicardial surface of the heart which were separated with great difficulty. Left internal mammary artery (LIMA) to LAD graft was fashioned on a beating heart with ease. But soon after establishment of flow to the LAD, the patient had an episode of VF and this was terminated by giving a DC shock. Intravenous amiodarone was started. Serum electrolytes were checked and were within normal limits. The patient was shifted to ICU in a stable condition. Just an hour after that the patient developed repeated episodes of VT leading to VF. A s the patient failed to respond to external shocks,

Authors' I nformation

1 Dr. Masoom Siraj, F RCS (Edin), Senior Consultant \& H ead, D epartment of Cardiac Surgery, Ibrahim Cardiac Hospital \& Research Institute, Shahbag, D haka.

${ }^{2}$ Lt Col (Retd) Dr. Md. Hamidur Rahman, FCPS, Senior Consultant \& Head, Department of Cardiac Anaesthesia, Ibrahim Cardiac Hospital \& Research Institute, Shahbag, D haka.

? Dr. F arzana Yasmin, M BBS, Assistant Registrar, D epartment of Cardiac Surgery, Ibrahim Cardiac H ospital \& Research Institute, Shahbag, D haka.

Correspondence: Dr. M asoom Siraj, M obile: 01711594096, E-mail: masoomsiraj@ hotmail.com 
he was re-opened in the ICU. A rrythmia was terminated after multiple DC shocks using internal paddles and internal cardiac massage. Thereafter an intra aortic balloon pump (IA BP) was inserted electively. The patient had a number of breakthrough episodes of VT, which, however responded to DC shocks and to intra-venous antiarrythmic drugs used at different time in the form of A miodarone, Lignocaine, Sotalol, and M agnesium Sulphate.

On the first postoperative day (POD) following CABG, patients generally exhibit multiple episodes of $V T$ and $V F$, almost always responding to cardioversion. In the present case, the patient was extubated on the same day. While the rhythm was sinus, patient's haemodynamics were absolutely normal and he communicated normally with the medical staff. He didn't have much of arrythmia during the night.

Early on morning of the second POD he developed arrythmia in the form of isolated ventricular ectopics. Patient was considered stable enough to the extent that IA BP was removed that day at 15:00 hrs. Unfortunately in the evening of the same day he started developing arrythmia in the form of VT and VF with greater frequency than before. All the medical management in the form of amiodarone, lignocaine, $\beta$-blocker, magnesium sulphate were used in varied combination as per medical advice. The same situation continued overnight with long duration of stability interspersed by bursts of arrythmia. On the third POD as the situation did not improve much, the patient was taken back to cathlab. Check CA G revealed a perfect LIMA to LAD graft. This opportunity was taken to put a stent on the nondominant small RCA. However this did not improve the situation. He continued to have periods of stability with periods of breakthrough $V T \& V F$.

Sadly the patient sustained another fatal episode of VT \& VF at 22.00 hrs. from which he failed to come out. During the entire duration opinion and advice was sought from cardiologists within and outside the hospital. Treatment was modified as and when needed according to advice given by the experts. It was an intense exercise for us in attempting to terminate the fatal arrythmia, despite the fact that we lost the patient.

\section{Discussion}

IVTA is an uncommon complication found in cardiac surgery. It is more common in those subset of patients having an episode of ischaemia, with poor ejection fraction and a dilated heart. All patients presenting as such need a detailed history including those of offending drugs if any, a physical examination, serum electrolytes and a work up for ischaemia.
In our case, episode of IVTA erupted after the element of ischaemia had been corrected by means of a graft. Patency of the same was later confirmed by repeat CA G and additionally a stent was given to a small nondominant RCA. Revascularization of an ischaemic heart is a proven way for controlling ventricular arrhythmia4,5. However very little is known how this reperfusion changes the electrophysiological substrate for the control of recurrent arrhythmia.6,7 In our case all electrolytes were within normal limits. Possibly abnormal circuits or action potential in relation to a damaged or stretched myocardium played the role. Reperfusion arrhythmia is known to be initiated at the interphase of damaged and newly revascularized tissue. All available modalities were used to suppress IVTA in our patient though without success.

M onomorphic VT is a result of reentry mechanism and an immediate cardiac defibrillation should be attempted. Further recurrences can be suppressed with medication. Available resources include amiodarone, ${ }^{8-12}$ lignocaine, verapamil, beta blockers \& magnesium sulphate all of which were used in our care at a time or other. Failure with such measures may bring about the need for bedside burst override pacing which was also employed for our patient.

If the above measures fail, IABP therapy is now an accepted method of terminating IVTA. Henson et al ${ }^{13}$ reported $86 \%$ improvement and $55 \%$ total resolution of post infarct VT/VF with IA B P. Bolooki et al ${ }^{14}$ reported a $50 \%$ resolution of IVTA with IA BP. How exactly IABP works is debatable. In ischaemic hearts it increases the coronary blood flow and rests the heart. However in a heart with a restored blood flow as in our case, it probably acts by reducing distension and workload of the left ventricle. Reduction in stretching and afterload of the left ventricle has shown a direct controlling effect of arrhythmia in animal models. ${ }^{15}$

What we were unable to offer to the patient was an electrophysiological study (EPS) followed by a possible implantable cardio defibrillator (ICD). This is something not available in our country on demand. Even if it was, the patient was unlikely to have been able to afford it. If electro-physiological maneuvers do not give benefit, patients in advanced western countries are put on ECM 0 followed by an emergency heart transplant. This is not feasible, practical or available in the context of a $B$ angladeshi centre.

One of the controversial issues in the genesis of arrhythmias in an ischaemic heart is the influence of acute ischaemic episode as opposed to chronic conditions. If ischaemia is identified as a trigger for arrhythmia short-lived or sustained, the restoration of 
blood flow to the myocardium should eliminate this event. A number of studies support that to be the case when ischaemia takes place in an acute setting. ${ }^{16,17}$ However Brugada and group showed, in chronic cases revascularization may not effectively suppress the proarrhythmia substrate. ${ }^{18}$ In their study easily inducible ventricular arrhythmia was documented in a very high percentage of patients despite coronary revascularization. Our patient could have been one of those belonging to the subset where revascularization and adjuvant treatment should have contained the recurrence of ventricular arrhythmia, but unfortunately the proarrythmia substrate persisted.

\section{Table I: Causes of Intractable Ventricular Tachy- Arryrthmia ${ }^{19}$ PRIMARY \\ Chronic M yocardial infarction \\ Dilated cardiomyopathy \\ Right Ventricular dysplasia \\ Primary Electrical Disorders \\ Status Post cardiac surgery \\ SECONDARY \\ Electrolyte abnormality \\ Severe hypoxia \\ A cute M yocardial infarction \\ Drugs}

Causes of IV TA as detailed in textbook is given in TableI. Primary causes are defined as those due to an abnormal, fixed substrate which is prone to arrhythmias. The key word here is "fixed". Secondary arrythmia are due to causes which are mostly reversible. In both the types mechanism includes reentry, automaticity and trigger. In order of frequency commonest cause is coronary artery disease, next is serum electrolyte disturbance followed by drugs.

Now if we look at our case in the light of the causes stated in standard literatures, all the secondary causes were eliminated during the course of our treatment. However, almost all the primary causes remained suspect and could have caused the fatal arrhythmias.

A $n$ interesting paper was published ${ }^{20}$ by a surgical team who had to undertake cardiac transplantation after all measures to control the ventricular fibrillation failed. They undertook pathological examination of the explanted heart. Their findings were as follows - chronic epicarditis, chronic myocarditis of the region of the AV node and arteriosclerosis. They concluded i) myocarditis of the smoldering type and/or fibrosis of the septum probably causes the intractable VT and VF II) intractable VT associated with cardiac arrest may be an indication for heart transplant. Our patient had chronic epicarditis as evidenced by dense pericardial adhesions. We can only postulate that he had a fixed substrate in the region of the septum which was trigerred by the revascularization.

We think it is important to identify which patient group has the potential to develop IV TA and prepare ourselves to the impending event. M ost patients will respond to the treatment modalities we offer. Going through the literatures we can see doctors in advanced western countries have also encountered similar stubborn IVTA. However those patients were fortunate enough to have the options of ICD, ECM $O$ and heart transplantation at their disposal. Sadly in Bangladesh our patients are not to be blessed with that.

\section{References}

1. Lo R, Hsia HH. Ventricular arrhythmias in heart failure. Cardiol Clin, 2008; 26:381- 403.

2. Cohen TJ. Invasive electrophysiologic techniques in the management of intractable ventricular arrhythmias. Cardiac Electrophysiol Rev 1999; 3:149-150.

3. Gillis A M. Intractable ventricular tachyarrhythmias: Immediate evaluation and management, role of pharmacologic therapy. Cardiac Electrophysiol Rev 1999; 3:145-148.

4. Alderman EL, Bourassa MG, Cohen LS, et al. Ten-year follow-up of survival and myocardial infarction in the randomized Coronary A rtery Surgery study. Circulation 1990; 82:1629-46.

5. The Veterans A dministration Coronary A rtery Bypass Surgery Cooperative Study Group. Eleven-year survival in the Veterans Administration randomized trial of coronary bypass surgery for stable angina. $\mathrm{N}$ Engl J M ed 1984; 311:333-9.

6. Geelen P, Primo J, Wellens F, B rugada P. Coronary artery bypass grafting and defibrillator implantation in patients with ventricular tachyarrhythmias and ischemic heart disease. Pacing Clin Electrophysiol 1999; 22:1132-9.

7. Ecker RR, Mullins CB, Grammer JC, et al. Control of intractable ventricular tachycardia by coronary revascularization. Circulation 1971; 44:666-70.

8. Kowey PR, Marinchak RA, Rials SJ, Bharucha DB. Intravenous antiarrhythmic therapy in the acute control of in-hospital destabilizing ventricular tachycardia and fibrillation. A m J cardiol 1999: 84:46R-51R.

9. Gorgels A PM, van der Dool A, Hofs A, et al. Comparison of procainamide and lidocaine in terminating sustained monomorphic ventricular tachycardia. A m J Cardiol 1996; 78:43-46.

10. Nasir N, Taylor A, Doyle TK, Pacifico A. Evaluation of intravenous lidocaine for the termination of sustained monomorphic ventricular tachycardia in patients with coronary artery disease with or without healed myocardial infarction, A m J Cardiol 1994: 74: 1183-1186.

11. Harrison EE, Lidocaine in pre-hospital countershock refractory ventricular fibrillation. A nn Emerg M ed 1981: 10: 410-423.

12. Guidelines 2000 for cardiopulmonary resuscitation and emergency cardiovascular care. Circulation 2000: 102 (Suppl 8).

13. Hanson EC, Levine $\mathrm{FH}, \mathrm{K}$ ay $\mathrm{HR}$, et al. Control of post-infarction ventricular irritability with the intraaortic balloon pump. Circulation 1980: 62 (Suppl 1): 1-30.

14. B olooki H. Intraaortic balloon pump for ventricular tachyarrhythmias. In: Bolooki H (ed). Clinical A pplications of Intraaortic Balloon Pump, Third Edition. A rmonk. N ew York: Futura Publishing Co., 1998 : pp. 336-339.

15. Hansen DE, Grig CS, Hondeghem LM. Stretch-induced arrhythmias in the isolated canine ventricle. Evidence for the importance of mechanoelectrical feedback. Circulation, $1990: 81: 1094-1105$.

16. Berntsen RF, Gunnes P, Lie M, et al. Surgical revascularization in the treatment of ventricular tachycardia and fibrillation exposed by exerciseinduced ischaemia. Eur Heart J 1993 : 14: 1297-303.

17. Rasmussen $\mathrm{K}$, Lunde $\mathrm{PI}$, Lie $\mathrm{M}$. Coronary bypass surgery in exerciseinduced ventricular tachycardia. Eur Heart] 1987; 8:444-8.

18. J oseph B rugada,L Luis M ont,A madeu B etriu,J aume M ulet.Coronary artery revascularization in patients with sustained ventricular arrhythmias in the chronic phase of a myocardial revascularization: effects on the electrophysiological substrate and outcome. I Am Col Cardiol.2001;37;529-533.

19. Todd, Cohen. Intractable ventricular tachyarrhythmia. Cardiac electrophysiology review. 1997; $1 / 2: 130-135$

20. Saroja B,B rian O,M aurice L.Pathological study of an explanted heart due to intractable ventricular fibrillation. J cardiovascular electrophysiology. 1992; Vol 3;Issue 5;437-441. 